\section{A BASIC program for computing Cronbach's accuracy components}

\author{
JOHN O. BROOKS III \\ Stanford University School of Medicine \\ Stanford, California \\ and \\ LAURA L. BROOKS \\ Rice University, Houston, Texas
}

\begin{abstract}
Accuracy is a measure of the extent of agreement between a rater's ratings and the ratings of experts or the responses of ratees. Cronbach (1955) argued that accuracy research should focus on components of accuracy rather than on an overall measure. However, complex calculations are required to compute Cronbach's accuracy components. A program is presented that provides a convenient way of computing all four components of accuracy (elevation, differential elevation, stereotype accuracy, and differential accuracy), as well as the associated correlation components, on an Apple Macintosh computer. The standard Marintosh interface is used to obtain all necessary information. The program will read data in a standard text file and will run faster on computers with math coprocessors.
\end{abstract}

The concept of accuracy can be applied in a variety of settings. In a typical experiment involving accuracy, ratees (e.g., employees, jobs, or patients) are rated on a number of items or dimensions. The resulting ratings are then compared to true scores, which derive from either the actual responses of the ratees or the average ratings of experts. Accuracy, then, is the extent to which the responses of a particular rater correspond to the true scores.

One way to measure a rater's overall accuracy is to use the sum of the squared differences between a rater's ratings and the true scores (e.g., Cronbach, 1955; Wiggins, 1973). Thus, if a rater rates $n$ ratees on $i$ items, the rating of ratee $k$ on item $m$ may be represented as $x_{k m}$, and the true score of ratee $k$ on item $m$ may be represented as $t_{k m}$. Overall accuracy may be expressed algebraically as in Equation 1.

$$
\text { Accuracy }=\frac{1}{n i} \sum_{k=1}^{n} \sum_{m=1}^{i}\left(x_{k m}-t_{k m}\right)^{2}
$$

In 1955, Cronbach argued that overall accuracy is misleading, because it is influenced by factors such as rater

Correspondence concerning this article should be addressed to John $O$ Brooks III, Department of Psychiatry and Behavioral Sciences (TD-114), Stanford University School of Medicine, Stanford, CA 94305-5490. Electronic mail may be sent to ID No. 72437,30 on CompuServe Information Services. biases that are not relevant to the assessment of accuracy. To resolve this problem, Cronbach proposed that accuracy was the sum of four components, which he termed elevation, differential elevation, stereotype accuracy, and differential accuracy. Because of the complexity of the formulae for these components, accuracy research has not flourished as it might have (Funder, 1987; Kenny \& Albright, 1987).

Research involving accuracy has generally supported Cronbach's (1955) argument that accuracy is made up of more than one component, in that the components of accuracy may be affected differently by experimental manipulations (e.g., Hahn \& Dipboye, 1988; Harvey \& Lozada-Larsen, 1988). Cronbach's accuracy components have been used in various research situations, such as job evaluation (Hahn \& Dipboye, 1988), person perception (DePaulo, Kenny, Hoover, Webb, \& Oliver, 1987), performance appraisal (Murphy, Garcia, Kerkar, Martin, \& Balzer, 1982; Pulakos, 1986), personality judgments (Funder, 1980), and sex-role predictions (Harackiewicz \& DePaulo, 1982).

Our purpose in this paper is to present a program that will provide an easy way to compute Cronbach's accuracy components on the Apple Macintosh computer. The operation of the program is best explained through a brief description of Cronbach's (1955) four components of accuracy and their formulae.

The first component, elevation (EL), summarizes the differences between the way a rater uses a scale relative to the true scores. Elevation is the squared difference between a rater's grand mean across all ratees and items $\left(\bar{x}_{.}.\right)$and the grand mean of the true scores across all ratees and items $(\bar{t}$.$) , as is illustrated in Equation 2 .{ }^{1}$

$$
\mathrm{EL}=\left(\bar{x}_{.}-\bar{t}_{.}\right)^{2}
$$

The remaining three components of accuracy-differential elevation, stereotype accuracy, and differential accuracy-can be written in terms of the variance of a difference and consequently include correlation terms (Cronbach, 1955). Cronbach pointed out that it is the correlational components of differential elevation and differential accuracy that reflect how sensitive a rater is to individual differences. This becomes apparent if the true scores and the rated scores are on different scales. In this situation, the four main components of accuracy may reflect nothing more than scaling differences, and the actual measures of accuracy would be the correlation components.

Differential elevation (DEL) represents the degree to which the deviations of a rater's mean rating of a ratee from the rater's mean of all ratees and items correspond 
to the deviation of the true scores for a ratee from the overall mean true score. Thus, differential elevation is the sum of the squared differences between two deviation scores and reflects the mean rating of a ratee. The formula for differential elevation is provided in Equation 3 , where $\bar{x}_{k}$. refers to the rater's mean for each ratee across all items, and $\bar{t}_{k}$, refers to the mean true score for each ratee across all items. The correlation component of DEL is the correlation between $\bar{x}_{k}$. and $\bar{t}_{k}$. Because these terms are the means for each ratee across items, the correlation represents a rater's ability to judge which ratees have the highest scores on the scale.

$$
\mathrm{DEL}=\frac{1}{n} \sum_{k=1}^{n}\left[\left(\bar{x}_{k}-\bar{x}_{. .}\right)-\left(\bar{t}_{k}-\bar{t}_{.}\right)\right]^{2}
$$

Stereotype accuracy (SA) describes a rater's ability to predict the average rating across all ratees for the items. Thus, stereotype accuracy depends on the rater's knowledge of the qualities of the group of ratees, or their stereotype. The formula for stereotype accuracy is provided in Equation 4, where $\bar{x}_{, m}$ refers to the average rating for each item across ratees, and $\bar{t}_{. m}$ refers to the average true score for each item across ratees. The correlational term of stereotype accuracy is the correlation between $\bar{x}_{, m}$ and $\bar{t}_{, m}$. These terms represent averages across ratees, and the correlation therefore represents the rater's ability to judge item means, or the group stereotype.

$$
\mathrm{SA}=\frac{1}{i} \sum_{m=1}^{i}\left[\left(\bar{x}_{. m}-\bar{x}_{.}\right)-\left(\bar{t}_{. m}-\bar{t}_{.}\right)\right]^{2}
$$

Finally, differential accuracy (DA) is an index of a rater's ability to predict differences among the ratees when all items are considered. Differential accuracy is computed with respect to each item and ratee and then averaged. Using the terms defined previously, differential accuracy is defined in Equation 5. The correlation pertaining to differential accuracy is the correlation between $\left(x_{k m}-\bar{x}_{k .}-\bar{x}_{. m}+\bar{x}_{. .}\right)$and $\left(t_{k m}-\bar{t}_{k .}-\bar{t}_{. m}+\bar{t}_{.}\right)$. This correlation between deviation scores is an index of a rater's ability to judge which ratees have the highest scores on an item.

$$
\begin{aligned}
& \mathrm{DA}=\frac{1}{n i} \sum_{k=1}^{n} \sum_{m=1}^{i}[\left(x_{k m}-\bar{x}_{k .}-\vec{x} \mathrm{x}_{. m}+\bar{x}_{.}\right) \\
&\left.-\left(t_{k m}-\bar{t}_{k}-\bar{t}_{\cdot m}+\bar{t}_{. .}\right)\right]^{2}
\end{aligned}
$$

\section{The Program}

The accuracy program provides a convenient way of computing the four components of accuracy (EL, DEL, $\mathrm{SA}$, and DA), as well as the three associated correlation components (DELr, SAr, and DAr). The program incorporates several machine language routines that increase the speed of the calculations (cf. Brooks, 1987). Machine language routines are "extensions" of BASIC that do not require the user to know machine language. The routines included with the program are in a machine language library called ExtraLib. These routines are part of the machine language library CLR MathStatLib, and they are distributed with the permission of Clear Lake Research (2476 Bolsover, Suite 343, Houston, TX 77005). The accuracy program will run faster on a Macintosh computer with a math coprocessor (e.g., a Macintosh SE/30, II, IIc, IIcx, IIci, and IIfx), but it can run on any Macintosh.

To compute accuracy, the ratings must be in an ASCII file arranged so that each rater's ratings are together. ${ }^{2}$ The ratings for the items for a given ratee should be on one line, and they should be separated by commas. As is shown in Figure 1A, the data for each ratee should be on a separate line, and the ratees must be sorted in the same order for each rater. Thus, if there are, say, 30 raters, 20 ratees, and 15 items, then the data should have 600 lines with 15 variables per line. The true scores should be in a separate ASCII file with one line per ratee, and the item scores should be separated by commas, as is shown in Figure 1B. If the files are set up in any other manner, accuracy will not be computed correctly. Because it is unclear how to deal with missing data in the computation of accuracy, all raters should have complete data.

After the program is started, the input screen illustrated in Figure 2 appears. The number of raters, ratees, and items must be entered in the appropriate boxes. The three buttons in the lower left portion of the screen are for file selection. The "Ratings" button and the "True scores" button allow the user to select the files that contain the ratings and true scores, respectively. The "Output file" button is used to provide the name of the ASCII file where the accuracy scores will be stored. File selection is accomplished through the standard Macintosh file dialog box.

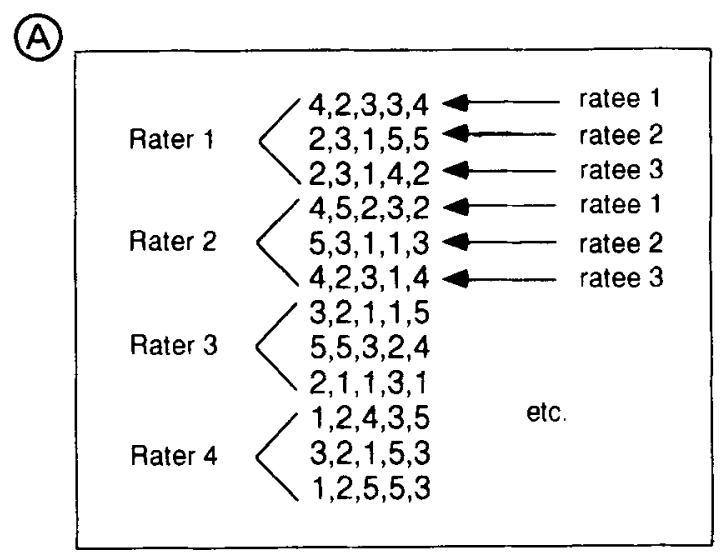

(B)

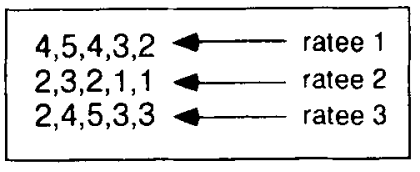

Figure 1. The ratings must be arranged as shown above. (A) An illustration of ratings from four raters for three ratees on five items. (B) A sample data set of true scores. There should be a carriage return at the end of each line, and the items on a line should be separated by commas. 


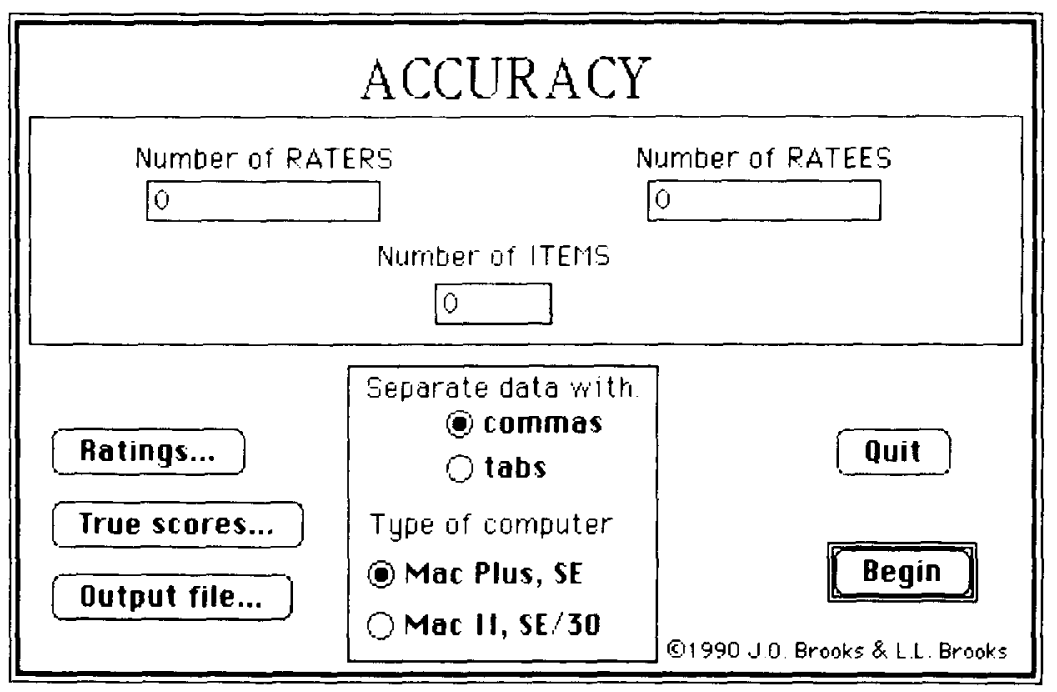

Figure 2. The screen used for entering the parameters of the dataset.

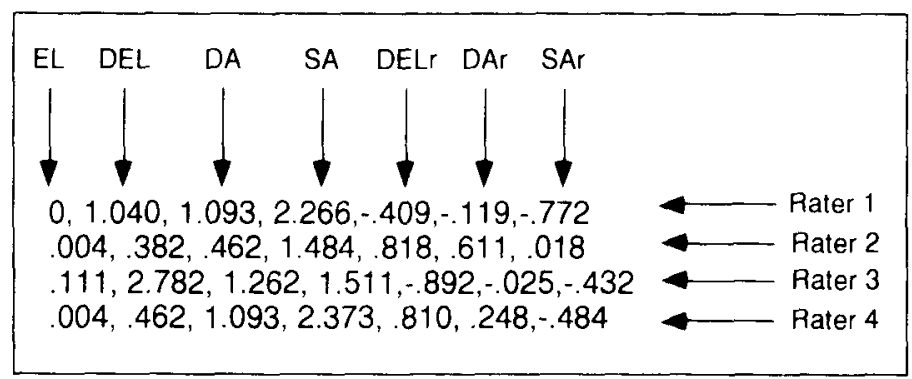

Figure 3. The accuracy data for each rater are on a separate line in the order indicated above. The accuracy scores were truncated to three decimal places for illustrative purposes. The program can delimit the output with either commas or tabs.

In contrast to the two input files, either commas or tabs may be used to delimit the data in the output file, depending on the requirements of the program that later reads the file. The button corresponding to the type of Macintosh should be chosen so that the program can take advantage of a math coprocessor if one is present. After all information has been entered, the computations can be started by clicking the "Begin" button or pressing the return key. If the "Begin" button is dimmed, all information has not yet been entered. The "Quit" button should be clicked to exit the program.

In the output file, which is illustrated in Figure 3, each rater's accuracy scores are placed on one line. Elevation is first, followed by differential elevation, differential accuracy, stereotype accuracy, and the correlation components of differential elevation, differential accuracy, and stereotype accuracy.

\section{Hardware and Software Requirements}

An Apple Macintosh computer with at least $1 \mathrm{MB}$ of RAM is required to run the program. A hard disk, though not required, will greatly increase the program's speed.
Microsoft QuickBASIC is not required for use of the compiled version of the program. The necessary machine language routines from CLR MathStatLib have been placed in a library called ExtraLib that is distributed with the program; users who have QuickBASIC and MathStatLib may simply enter the program from the listing and change the LIBRARY statement to LIBRARY "MathStatLib." Included with the program disk are a sample file of data on which the accuracies have been computed and stepby-step instructions.

\section{Availability}

An $800 \mathrm{~K}$ disk containing the program listing, the compiled version of the program, the machine language routines, and the sample data may be obtained by sending $\$ 6$ to the first author. The first author may be contacted on Compuserve Information Services at ID No. 72437,30.

\section{REFERENCES}

Brooks, J. O., III. (1987). Enhancing and degrading visual stimuli. Behavior Research Methods, Instruments, \& Computers, 19, 260-269. 
Cronbach, L. J. (1955). Processes affecting scores on "understanding of others" and "assumed similarity." Psychological Bulletin, 52, 177-193.

DePaulo, B. M., Kenny, D. A., Hoover, C. W., Webb, W., \& OLVER, P. V. (1987). Accuracy of person perception: Do people know what kinds of impressions they convey? Joumal of Personality \& Social Psychology, 52, 303-315.

Funder, D. C. (1980). On seeing ourselves as others see us: Self-other agreement and discrepancy in personality ratings. Journal of Personality, 48, 473-493.

FUNDER, D. C. (1987). Errors and mistakes: Evaluating the accuracy of social judgment. Psychological Bulletin, 101, 75-90.

HAHN, D. C., \& Dipboye, R. L. (1988). Effects of training and information on the accuracy and reliability of job evaluations. Joumal of Applied Psychology, 73, 146-153.

Harackiewicz, J. M., \& DePaulo, B. M. (1982). Accuracy of person perception: A component analysis according to Cronbach. Personality \& Social Psychology Bulletin, 8, 247-256.

HARVEY, R. J., \& Lozada-LARSEN, S. R. (1988). Influence of amount of job descriptive information on job analysis rating accuracy. Journal of Applied Psychology, 73, 457-461.

KenNy, D. A., \& ALBRIGHT, L. (1987). Accuracy in interpersonal perception: A social relations analysis. Psychological Bulletin, 102, 390-402.

Murphy, K. R., Garcia, M., Kerkar, S., Martin, C., \& Balzer, W. K. (1982). Relationship between observational accuracy and accuracy in evaluating performance. Journal of Applied Psychology, 67, 320-325.
Pulakos, E. D. (1986). The development of training programs to increase accuracy with different rating tasks. Organizational Behavior \& Human Decision Processes, 38, 76-91.

Wiggins, J. S. (1973). Personality and prediction: Principles of personality assessment. Reading, MA: Addison-Wesley.

\section{NOTES}

1. Cronbach (1955) abbreviated the accuracy components as $E L^{2}$, $\mathrm{DEL}^{2}, \mathrm{SA}^{2}$, and $\mathrm{DA}^{2}$. This is somewhat confusing, however, for these "squared" components are, in fact, the accuracy components, and they can be summed to yield overall accuracy. For this reason, our abbreviations of the accuracy components do not include the exponents.

2. For persons unfamiliar with various file types, an ASCII file is a sort of "universal" file format. Virtually every Macintosh application (e.g., Microsoft Word, StatView, Microsoft Excel, SYSTAT, etc.) can save files as "text only." Similarly, most applications on other computers (e.g., IBM PCs, mainframes) can create ASCII files that can be transferred to a Macintosh. However, not all applications delimit variables with commas. For this reason, a BASIC program is provided on the distributed disk (with brief instructions) that will convert a tabdelimited file, such as those created with Excel, to a comma-delimited file.

(Manuscript received February 5, 1990; revision accepted for publication April 30, 1990.) 